\title{
Polymeric compositions having near IR photoconduction
}

\author{
N.A. Davidenko ${ }^{1}$, N.G. Kuvshinsky ${ }^{1}$, D.A. Melenevsky ${ }^{1}$, N.A. Derevyanko ${ }^{2}$, \\ A.A. Ishchenko ${ }^{2}$, A.V. Kulinich ${ }^{2}$, O. Neilands ${ }^{3}$, M. Plotniece ${ }^{3}$ \\ ${ }^{1}$ Taras Shevchenko Kiev National University, Kyiv, Ukraine \\ ${ }^{2}$ Institute of Organic Chemistry, National Academy of Sciences of Ukraine, Kyiv, Ukraine \\ ${ }^{3}$ Riga Technical University, Riga, Latvia
}

\begin{abstract}
Peculiar properties of carrier photogeneration in the polymer compositions based on polystyrene containing substituted tetrathiafulvalenes - electron donors, 2,4,5,7-tetranitro9-fluorenone - acceptor of electrons, cationic polymethine dye - tetrafluoroborate 1,3,3trimethyl-2-[3-(1,3-dihydro-1,3,3-trimethyl-2H-2-indolyliden)-1-propenyl]-3H-indolium and neutral merocyanine - 5-[6-(1,3-dihydro[1,3,3-trimethyl-2H-indol-2-yliden)-4-phenyl-3,5-(ethylene)-hexa-2,4,6-trien-1-yl] thiobarbituric acid - sensitizers. The photoconductivity in the dye absorption range increases, when the cation dye changed to the neutral, mostly due to mobile carrier with both charges sign photogeneration, and the activation energy of the photoconductivity current decrease. The decrease of photoconduction current activation energy can be related to the organic charged counterion generation in the neutral dye case, and the uncoloured counterion retains more strongly the opposite charge on the photogenerated organic ion in the cation dye case.
\end{abstract}

Keywords: polymeric compositions, tetrathiafulvalenes, organic dyes, carrier photogeneration, electron-hole pairs.

Paper received 15.12.03; accepted for publication 17.06.04.

\section{Problem definition}

To create new materials possessing photoconduction properties in the near IR range is a very actual problem for the solar energy photoelectric convert [1-3]. Along with non-organic semiconductors, it seems promising to use the organic polymers and their compositions [3]. The latest material should meet the following requirements [3]: the high absorption coefficient in the range 700$900 \mathrm{~nm}$, high carrier photogeneration quantum yield, high non-equilibrium carrier mobility, and the absence carrier capture in the film volume and nearby to the electric contacts to avoid its accumulation and conduction current limiting.

In a general case, photoconduction of the polymer compositions is provided by three types of molecules; donors, acceptors, and carrier photogeneration sites, which placed in them [4]. When the absorb the light quantum by the photogeneration site it can be the dye molecule, the organic molecule compound with the intermolecular CT-complex, then this site switches from the ground state to the exited state. The excited state of the photogeneration site is characterized by the bounding unoccupied orbital, with one electron present on HOMO and LUMO. The carrier photogeneration takes place as a result of the intermolecular electron transfer: an electron can switch from the LUMO exited photogeneration site to LUMO of the molecule with the electron-seeking properties; and the electron can switched to HOMO photogeneration site from the valence orbital with electron-donor properties. If the donor molecule has an ionization potential $I_{g d}$ (HOMO energy is proportional to it), and the acceptor molecule has an affinity energy to the electron $A_{e a}$ (LUMO energy is proportional to it) [5], then the photogeneration site molecules are selected accordingly to the following conditions: $\left|I_{g c}\right|>\left|I_{g d}\right|$, $\left|A_{e c}\right|<\left|A_{e a}\right|$, where $I_{g c}$ and $A_{e c}$ are the ionization potential and affinity energy for the electron. The realization of these conditions defines, that after the light quantum absorption in the molecular photogeneration site, and in the intermolecular electron transitions the electron-hole pair (EHP) is generated. If EHP is generated from the electro-neutral or bipolar (intraion) molecular site, then it consists of the donor cation-radical and the photo. generation site anion-radical, or photogeneration site cation-radical and the acceptor anion-radical, donor cation-radical and the acceptor site anion-radical. EHP dissociation results in appearance of free carrier.

The aim of this work was to investigate the photophysical properties of the polymer composition films with 


\section{N.A. Davidenko et al.: Polymeric compositions having near IR photoconduction}

various organic donors, acceptors and photogeneration sites - neutral and ion dyes. The solution of this problem will give the key to creation of new effective photoconducting materials for the near IR range.

\section{Subjects of inquiry}

The polystyrene (PS) was used as a polymer basis. The monomer epoxypropylcarbazole (EPC) - as donor molecules<smiles>c1ccc2c(c1)c1ccccc1n2CC1CO1</smiles>

and tetrathiafulvalenes, D1, D2.



D1<smiles>CC1=C(C)SC(=C2CC(C(C)C)=CS2)S1</smiles>

D2

We used 2,4,5,7-tetranitro-9-fluorenone as an acceptor (TENF)<smiles>O=C1c2cc([N+](=O)[O-])cc([N+](=O)[O-])c2-c2c1cc([N+](=O)[O-])cc2[N+](=O)[O-]</smiles>

The photogeneration sites were CT-complexes between EPC, D1, D2 and TENF, or HITC and HITB5<smiles>CN1/C(=C\C=C\C2=[N+](C)c3ccccc3C2(C)C)C(C)(C)c2ccccc21</smiles>

HITC<smiles>CN1C(=CC=C2C=CC(C=C3C(=O)NC(=S)NC3=O)=C2c2ccccc2)C(C)(C)c2ccccc21</smiles>

HITB5

The quantum-chemical calculations of the investigated compounds were performed by the semi-empirical method AM1 with the standard parameter sets [6]. The calculations were performed according to the interaction of one-fold excited configurations, due to all possible electron transitions from three upper HOMOs to three lower LUMOs. Molecular geometry optimization was previously done.

The samples for investigations were prepared by sprinkling onto glass substrates or substrates with the thin conducting layer $\mathrm{SnO}_{2}: \mathrm{In}_{2} \mathrm{O}_{3}$ using toluene solutions of PS with the investigated additive compounds. The substrates were dried out in the oven at the temperature $+80^{\circ} \mathrm{C}$ for 4 hours. The concentration of donor molecules with respect to PS mass was changed within the range 0 to $30 \%$, TENF -0 to $20 \%$, dyes - 0 to $1 \%$. The thickness of prepared polymer films was up to $1-2 \mu \mathrm{m}$. The sandwichstructure samples illuminated the monochromatic light were used for the dark-current $\left(j_{t}\right)$ and photocurrent $\left(j_{P H}\right)$ measurements. The light intensity $(I)$ in the range $0.2-5 \mathrm{~W} / \mathrm{m}^{2}$ was changed by neutral light filters. The electric field $(E)$ in the polymer film with influence of the electric voltage to the $\mathrm{Al}$ and $\mathrm{SnO}_{2} \mathrm{In}_{2} \mathrm{O}_{3}$ contacts was being changed within the range $(1 \ldots 20) \cdot 10^{7} \mathrm{~V} / \mathrm{m}$. The measurements of $j_{t}$ and $j_{P H}$ were done in the temperature range 290 to $355 \mathrm{~K}$, the thermostat with the thermo-element and the optical window was used. The activation energies of electroconductivity $\left(W_{t}\right)$ and photoconductivity $\left(W_{P H}\right)$ were determined from $j_{t}$ and $j_{p h}$ temperature dependences.

\section{Experimental results and discussion}

The PS films without additive compounds don't absorb visible light (with wave lengths longer than $400 \mathrm{~nm}$ ), and they don't show the photoconduction effect in the sandwich-structure samples over the whole investigated electric field region. In the PS+TENF films, the long-wave edge of the fundamental absorption becomes visible, for $\lambda>400 \mathrm{~nm}$ (Fig. 1, curve 1 ). The PS films with $1-30 \%$ of the EPC by their mass, without additives don't absorb the visible range light in the sandwich-structure samples, and don't show the photoconduction effect. The PS films with $1-30 \%$ of the D1 by mass and PS films with $1-30 \%$ $\mathrm{D} 2$ by mass absorb in the $\lambda>400 \mathrm{~nm}$ region, which was determined from the boundary of intrinsic absorption of D1 and D2 (curve 2 and 3, Fig. 1). $\kappa$ value increases in proportion to the concentration increase, while the form of the absorption band doesn't change. The latter statement corresponds to a small departure of EPC, D1, D2 molecules to aggregation in PS films. However, as compared with those films in the PS with $1-30 \%$ of the EPC, D1, D2 by mass $+1-20$ mass \% of TENF in optical absorption spectra, the new thick bands appear. value increases when TENF concentration does, but the absorption band sharp doesn't change.

This implies that these absorption bands are related with the creation of the CT-complex between EPC, D1, D2 donor and TENF acceptor. The bathochromic shift of the intrinsic absorption edge inherent to the films in the point where EPC have been changed to D1 and D2 is 


\section{N.A. Davidenko et al.: Polymeric compositions having near IR photoconduction}

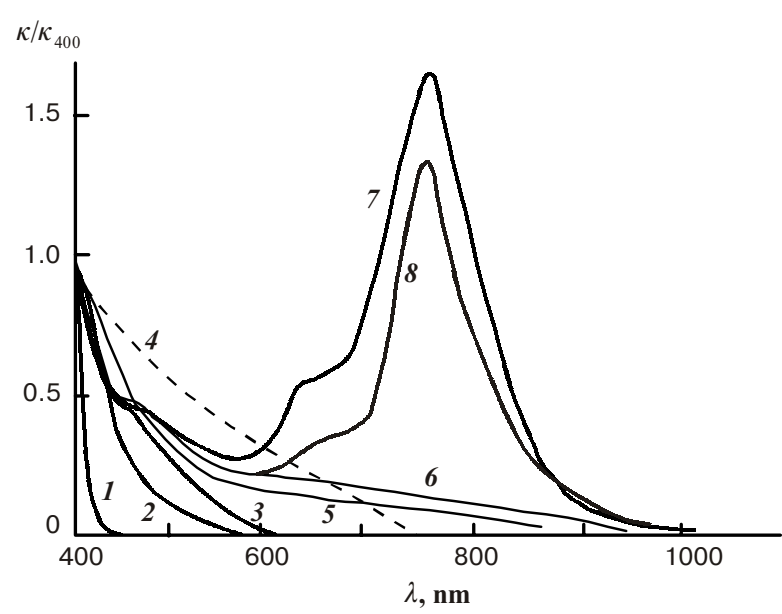

Fig. 1. The absorption spectra of he PS $+10 \%$ TENF by mass (curve 1 ), PS $+20 \% \mathrm{D} 1$ by mass (2), PS $+20 \% \mathrm{D} 2$ by mass (3), PS $+20 \%$ EPC by mass $+10 \%$ TENF by mass (4), PS $+20 \%$ D 1 by mass $+10 \%$ TENF by mass (5), PS $+20 \% \mathrm{D} 2$ by mass $+10 \%$ TENF by mass $(6)$, PS $+20 \% \mathrm{D} 2$ by mass $+10 \%$ TENF by mass $+1 \%$ HITC by mass (7), PS $+20 \% \mathrm{D} 2$ by mass $+10 \%$ TENF by mass $+1 \%$ HITB 5 by mass (8). $\kappa_{400}-$ the absorption factor for the $\lambda=400 \mathrm{~nm}$.

defined by $I_{g d}$ decrease in this line of donors and the light quantum energy decrease $\left(h v_{C T}\right)$, which is necessary for the full transfer of electrons from donors to acceptors in CT-complex. It is confirmed by the comparison of the experimentally determined values $I_{g d}$ for EPC, D1, D2, and from the results of the quantum-chemical calculations of HOMO and LUMO energies.

The results of our quantum-chemical calculations of these energies, which use donors, acceptor and dyes, are shown in Fig. 2. We would note that these results can't be used to determine numerical quantities with a high accuracy of ionization potentials and the affinity energy for electrons in the investigated compounds. But in workmanlike manner, the equation (1) is held if some dopant substitutes in PS films fully regular. One can see that in the EPC, D1, D2 line, the $\left|I_{g d}\right|$ decrease and to the greater extent corresponds to the experimental data when the method AM1 is used.

In the sandwich-structure samples with the PS+ $+(1-30) \%$ EPC,D1,D2 by mass $+(1-20) \%$ TENF by mass, the photocurrent for the absorption spectral regions respected to the CT-complex was detected. If EPC substitutes to D1 and D2, this region has switched bathoromicaly. When $E$ and $I$ are constants, $j_{P H}$ value increases if the donor and/or acceptor concentration increases, and it doesn't depend on polarity of the applied electric voltage, but it decreases with increasing the wavelength in proportion to $\lambda$ change. Besides, the plot of $j_{P H}$ depends on $I$ is the straight line, and the dependences of $j_{P H}$ on $E$ can be approximated by the straight lines in $\lg j_{P H}-E^{1 / 2}$ coordinates (Fig. 3) using the value of line slope tangents it is possible to calculate the Pool-Frenkel constant, and its value is $4.5 \cdot 10^{-5} \mathrm{eV} \cdot \mathrm{V}^{-1 / 2} \cdot \mathrm{m}^{1 / 2}$, which is close to the theoretical one [4,7]. The dark current and the photocurrent values increase with increasing temperature. It means that the photoconductivity of the samples caused by the carrier bulk photogeneration and their transport inside the polymer films. The carrier photogeneration mechanism is similar to that in poly-N-epoxypropylcarbazole with TENF films: EHP phogeneration sites are CT-complexes [4,7], EHP dissociation in the external electric field takes place by dint of the hole intermolecular switches by EPC, D1, D2 donor molecules, and electrons by TENF acceptor molecules.

In the sandwich-structure films with donors, TENF and dyes, the dark current and the photocurrent are much larger than in the similar samples without dyes (Fig. 3). However, in the samples with the PS+(1-30) mass \% EPC $+(1-20)$ mass \% TENF +1 mass \% HITC, HITB5 films, in the dye absorption ange (for $\lambda>700 \mathrm{~nm}$ ) the photocurrent is not essential, but increases when EPC is changed to D1 or D2. Besides, the photocurrent in the dye absorption range increases when HITC is changed to HITB5, though the film factor with HITB5 films is smaller than with HITC (Fig. 1). Values of the dark current and photocurrent don't depend on polarity of the applied voltage. The most essential value of rise rate, the photocurrent relaxation, and the characterizing velocity of the non-equilibrium carrier motion in the electric field [4], are in the film-samples with the D2, TENF and HITB5 (Fig. 4). The plots of dependences for $j_{t}, j_{P H}$ against $T$ in the temperature range $290-355 \mathrm{~K}$ are linear in the Arrhenius coordinates. The calculated values of affinity energy $W_{t}$ and $W_{P H}$ are close to each other. For example, as for the sandwich-structure samples with PS + 20 mass $\%$ D $2+10$ mass $\%$ TENF +1 mass $\%$ HITB 5 films when the electric field is small, the values of $W_{t}$ and

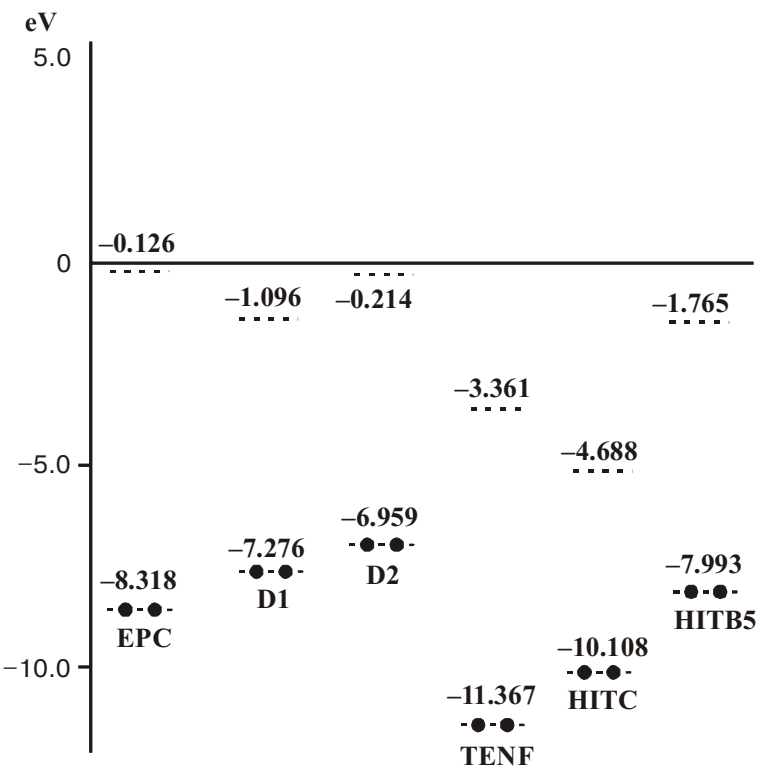

Fig. 2. The molecule HOMO (solid horizontal line) and LUMO energies (dash horizontal line). 


\section{N.A. Davidenko et al.: Polymeric compositions having near IR photoconduction}

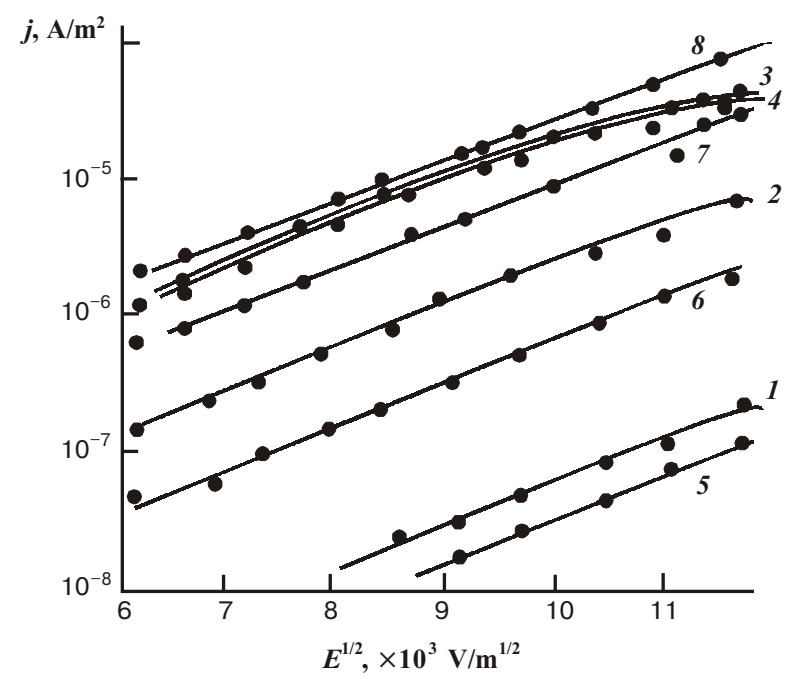

Fig. 3. The dependence of the darkcurrent (1-4) and photocurrent (curves 5-8) in the sandwich-structure samples with the PS $+20 \%$ EPC by mass $+10 \%$ TENF by mass $+1 \%$ HITB 5 by mass $(1,5)$, PS $+20 \%$ D 1 by mass $+10 \%$ TENF by mass $+1 \%$ HITC by mass $(2,6)$, PS $+20 \%$ D 2 by mass $+10 \%$ TENF by mass $+1 \%$ HITB 5 by mass $(3,7)$ films, $\lambda=760 \mathrm{~nm}, T=293 \mathrm{~K}$.

$W_{P H}$ are $0.23 \pm 0.01 \mathrm{eV}$. In the samples with: $\mathrm{PS}+$ +20 mass $\% \times \mathrm{D} 2+10$ mass $\%$ TENF +1 mass $\%$ HITC films these values are $0.45 \pm 0.02 \mathrm{eV}$. According to the stated above these films for $\lambda>700 \mathrm{~nm}$ the photoconductivity and electroconductivity are mainly caused by generation of bilk carriers due to light absorption by dye molecules. HITB5 and HITC molecules are the non-equilibrium carrier photogeneration sites, and the carries thermo-emf generation. The photoconduction and electroconduction increase in the polymer films which contain donors EPC, D1, D2 and HITC shows that energy differences $\left|I_{g c}\right|-\left|I_{g d}\right|$ increase, and the conditions to generate mobile carriers (holes) become better for such number of donors. Fig. 2 shows that the condition $\left|I_{g s}\right|>\left|I_{g d}\right|$ that is necessary for the hole generation from the excited molecules of dye is satisfied done for HITC and HITB5 with studied donors, and the condition $\left|A_{e c}\right|<\left|A_{e a}\right|$, that means the requirement for electron generation, is fulfilled only for HITB with the studied TENF acceptor. It means that, contrary to HITC, HITB5 molecules can be the photogeneration sites of both holes and electrons. Above said was confirmed in an experimental way; the photocurrent in PS samples with D1, D2 films, containing HITB5 and TENF (Fig. 4 curve 6) under the same conditions, is higher than the sum of photocurrents in the similar sample, but containing HITB5 or TENF (Fig. 4). The photocurrent in PS samples with D1 and D2 containing HITC and TENF (Fig. 4) is manly close to the sum of photocurrents in similar samples, but containing either HITC or TENF (Fig. 4).

However in the films with HITB5, the photoconduction increase as compared to similar films but containing HITC is caused by another reason, $W_{t}$ and $W_{P H}$ increase takes place when HITB5 is changed with HITC. In the model of the second stage of carrier photogeneration via excitation EHP and their dissociation, the value WPH calculated from the dependence of $j_{P H}$ on $T$, when $E$ is small, may be equaled to the Coulomb interaction energy between the electron and hole in EHP: $W_{0 P H}=q^{2} / 4 \pi \varepsilon_{0} \varepsilon r_{0}$ where $q$ is the electron charge, $\varepsilon_{0}$ - the permittivity, $\varepsilon-$ the electric constant of the medium, $r_{0}$ - the distance between the opposite charges in the EHP. For simplicity, the non-excited dye molecule forms can be represented as (D-A) - for HITB5, (D-D+..An ${ }^{-}$- for HITC, where $\mathrm{An}-$ is the uncoloured counterion of the ion dye. Adduced below is EHP generation in PS with D2, TENF, HITB5 films and in PS with D2, TENF, HITC films, with proper reactions of the electron transfer.

$$
\begin{aligned}
& \mathrm{D} 2+(\mathrm{D}-\mathrm{A})+\mathrm{TENF} \stackrel{h v}{\longrightarrow} \\
& \stackrel{h v}{\longrightarrow} \mathrm{D} 2+\left(\mathrm{D}^{+}-\mathrm{A}^{-}\right)^{*}+\mathrm{TENF} \rightarrow \\
& \rightarrow \mathrm{D}^{\bullet+}+\left(\mathrm{D}^{-\mathrm{A}^{-}}\right)+\mathrm{TENF} \rightarrow \\
& \rightarrow \mathrm{D} 2^{\bullet+}+(\mathrm{D}-\mathrm{A})+\mathrm{TENF}^{\bullet}, \\
& \mathrm{D} 2+\left(\mathrm{D}-\mathrm{D}^{+} \ldots \mathrm{An}^{-}\right)+\mathrm{TENF} \stackrel{h v}{\longrightarrow} \\
& \stackrel{h v}{\longrightarrow} \mathrm{D} 2+\left(\mathrm{D}^{+}-\mathrm{D} \ldots \mathrm{An}^{-}\right)^{*}+\mathrm{TENF} \rightarrow \\
& \rightarrow \mathrm{D} 2^{\bullet+}+\left(\mathrm{D}^{\bullet}-\mathrm{D} \ldots \mathrm{An}^{-}\right)+\mathrm{TENF} .
\end{aligned}
$$

Here we took into account that just after the light quantum absorption, the electron density redistribution

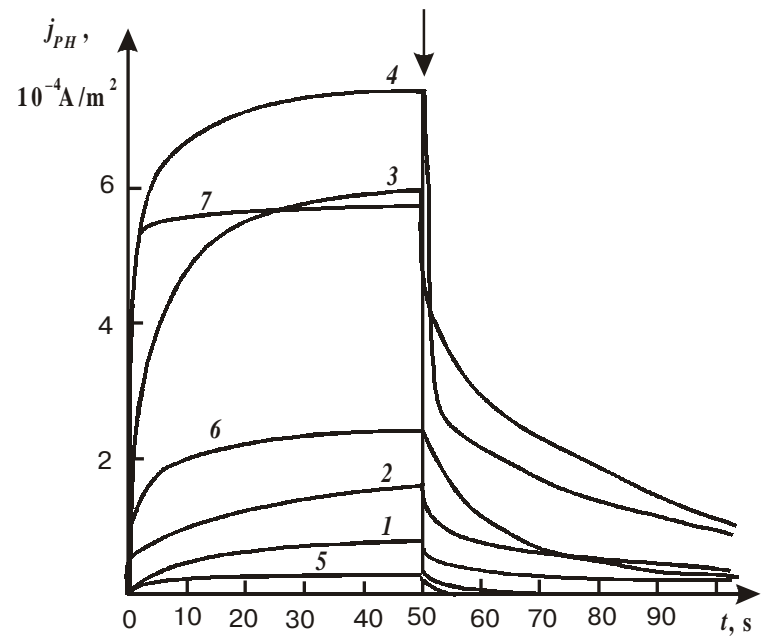

Fig. 4. The dependence of $j_{P H}$ on the time $t$, after illumination with $\lambda=760 \mathrm{~nm}$ in the sandwich-structure samples with PS $+20 \% \mathrm{D} 1$ by mass $+10 \%$ TENF by mass (1), PS $+20 \% \mathrm{D} 2$ by mass $+10 \%$ TENF by mass (2), PS $+20 \% \mathrm{D} 2$ by mass $+1 \%$ HITC by mass (3), PS $+20 \% \mathrm{D} 2$ by mass $+10 \%$ TENF by mass $+1 \%$ HITC by mass (4), PS $+20 \%$ D 2 by mass $+1 \%$ HITB 5 by mass (5), PS $+20 \%$ D 2 by mass $+10 \%$ TENF by mass $+1 \%$ HITB 5 by mass (6) films. The vertical arrow corresponds to the time moment when the illumination was switched on. $E=1.1 \cdot 10^{8} \mathrm{~V} / \mathrm{m}\left(I-4, I=1 \mathrm{~W} / \mathrm{m}^{2}\right) . T=293 \mathrm{~K}$, the thickness of the polymer film is $1.5 \mu \mathrm{m}$. 


\section{N.A. Davidenko et al.: Polymeric compositions having near IR photoconduction}

inside the dye molecules takes place, with generation of their excited states: $\left(\mathrm{D}^{+}-\mathrm{A}^{-}\right)^{*}$ for HITB5, and $\left(\mathrm{D}^{+}\right.$ $\left.-\ldots \mathrm{An}^{-}\right)^{*}-$ for HITC. The relaxation of the excited phototogeneration sites, results in generation of the carriers. In film PS samples with D2, TENF, HITB5, from the excited molecule state the hole appears, which correspond to the anion-radical D2 (D2 $\left.{ }^{\circ}\right)$ and anion-radical HITB5 $\left(\mathrm{D}^{-}-\mathrm{A}^{-}\right)$, appearance and can give one electron to TENF molecule with anion-radical $\left(\mathrm{TENF}^{\bullet}{ }^{-}\right)$generation. In film PS samples with D2, TENF, HITC, when the cation dye molecule absorbs light, only the hole $\left(\mathrm{D} 2^{\cdot+}\right)$ and anion-radical $\left(\mathrm{D}^{*}-\mathrm{D} \ldots \mathrm{An}^{-}\right)$are generated.

The schemes (1), (2) shows the difference between neutral and ion dyes for carrier photogeneration. It means that in the reactions (1), the electrically charged pairs of the molecular organic radicals, these structures are used for the carrier and photogeneration site transport, in the reactions (2) - the electrically charged pairs of the molecular organic radicals, and its used for the carries and the non-colored counterion transport. So, as on the second stage of the photogeneration, during charge pairs dissociation, the mobile carrier move off from the opposite charged sites. It is necessary to regard as in the films with the ion dyes, unlike the films with neutral dyes, the photogeneration activation energy $W_{0 P H}$ is determined by the Coulomb interaction energy of mobile carriers with the electrically charged non-colored counterion, and in this case the $r_{0}$ value corresponds to the distance between the photogenerated carrier and the counterion.

The assumption about the deference between neutral and ion dyes, as a photogeneration sites, can give the explanation for $W_{0 P H}$ increase when HITB5 is changed with HITC. This explanation is as follows. In PS with donors, acceptors and HITB5 films, the spatial distribution of the dopant molecules are isotropic, and there are no special impurity ions. Therefore, under the carrier photogeneration from HITB5 molecules, the radius-vector direction between EHP charges can be isotropic and equiprobable for any separately taken molecule of the photogeneration site. In PS films with donors, acceptors and HITC, the spatial distribution of the dopant molecules is also isotropic, but the non-colored ions are present there, and their concentration are equal to the concentration of photogeneration sites. The molecules of ion organic dyes in PS films can be as a parted and as a contacted charge pairs. It means that in EHP generation when the carrier leaves $\left(\mathrm{D}^{+}-\mathrm{D} \ldots \mathrm{An}^{-}\right)^{*}$ to the donor molecule, the best direction will be that with the localized nearest counterion $\mathrm{An}^{-}$. In the latter case, the distance $r_{0}$ should be smaller, and the Coulomb interaction energy between the opposite charges - higher. We should mark, that the electric charge in the uncoloured counterion of the ion dye is localized much, than the mentioned energy increase higher in compare to the same energy but in the EHP generation by reaction (1) [8]. Also from the dye molecules in the carrier thermogeneration, the opposite charge pairs are generated, then $W_{t}$ and $W_{P H}$ values are similar between each one, but different for the different dye types.

\section{Conclusions}

The tetrathiafulvalenes can be used as donors of electrons in IR photoconducting polymer films. To increase its solubility and decrease aggregation, which reduce to the polymers compound increasing, it is necessary volumetrically substitutes, like rich radicals (molecules of the D1 and D2) bring in to the donor molecules. They are laced with the polymer chain, impeding the aggregation. The organic dyes are used preferably as carrier photogeneration sites in IR spectral range. So, it is necessary to take into account that the cation dyes with the high donor concentration used in polymer films lead to the monopolar character of photoconductivity, caused by more effective photogeneration of holes. Slower electrons that stay in the cation dye molecules after EHP photogeneration are effective carrier strapping and recombination sites. The electric charge of the uncoloured counterions influences on the mobile carrier photogeneration process in the ion dye films, which work as the carrier photogeneration sites. Closely to the gegenions on the first stage of the photogeneration, the photogenerated carries are localized. The photogeneration and electroconduction activation energies are defined in the previous charge pairs with the electrostatic interaction. As the distances between atoms in the studied pairs can be small, the experimental data describing the influence of the counterion type to the photoconductivity spin effects, becomes explicable [9]. The photogeneration of both signs carries, realized in these polymer films, which contain donors, acceptors and neutral dyes. The high photoconductivity is reached due to the mobile electrons and holes, which are generated in the EHP photogeneration, its recombination in the dyes molecules is extremely difficult. In these films with the neutral dyes, the mobile carries photogeneration takes place in the electrostatic field of the ionized photogeneration site, then the spatial distribution of the radius-vector between EHP charges is an isotropic, and the electrostatic interaction energy between EHP charges can be smaller than in the ion dyes case, under the same conditions.

\section{References}

1. J. Rostalski, D. Meissner // Solar Energy Mater, Solar Cells, 61, P. 87 (2000).

2. J.-M. Nunzi // C. R. Physique, 3(1) P. 1 (2002).

3. N.A. Davidenko, A.A. Ishchenko // Theoretical and Experimental Chemistry, 38(2), P.88 (2002).

4. N.G. Kuvshinsky, N.A. Davidenko, V.M. Komko. Physics of amorphous molecular semiconductors. Kiev: Lybid', 1994.

5. D. Danovich, Y. Apeloig, S. Shaik // J. Chem. Soc., Perkin Transactions, N3, P.321 (1993).

6. J.J. P.Stewart // J. Computer-aided, N4, P.1 (1990).

7. M. Pope, C.E. Swenberg, Electronic Processes in Organic Crystals. Oxford: Clarendon Press, 1982.

8. N.G. Kuvshinsky, N.A. Davidenko, V.V. Reshetnyak, L.I. Savransky, V.L. Sheptun // Chem. Phys. Lett., 165, P. 323 (1990).

9. N.A. Davidenko, A.A. Ishchenko // Chem. Phys, 247, P. 237 (1999). 